2007-6

\title{
Evaluation of the IDI-MRSA assay on the SmartCycler real-time PCR platform for rapid detection of MRSA from screening specimens
}

\author{
Celine Herra \\ Technological University Dublin, celine.herra@tudublin.ie
}
A. S. Rossney
St. James's Hospital, Dublin
M. Fitzgibbon
St. James's Hospital,Dublin

See next page for additional authors

Follow this and additional works at: https://arrow.tudublin.ie/scschbioart

Part of the Medicine and Health Sciences Commons

\section{Recommended Citation}

Herra, C. et al. (2007) Evaluation of the IDI-MRSA assay on the SmartCycler real-time PCR platform for rapid detection of MRSA from screening specimens, Eur J Clin Microbiol Infect Dis vol. 26, pp.459-466 DOI 10.1007/s10096-007-0303-7

This Article is brought to you for free and open access by the School of Biological Sciences at ARROW@TU Dublin. It has been accepted for inclusion in Articles by an authorized administrator of ARROW@TU Dublin. For more information, please contact arrow.admin@tudublin.ie, aisling.coyne@tudublin.ie,gerard.connolly@tudublin.ie.

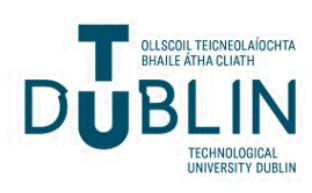




\section{Authors}

Celine Herra, A. S. Rossney, M. Fitzgibbon, P. Morgan, and B. O'Connell 


\title{
Evaluation of the IDI-MRSA assay on the SmartCycler real-time PCR platform for rapid detection of MRSA from screening specimens
}

\author{
A. S. Rossney • C. M. Herra • M. M. Fitzgibbon • \\ P. M. Morgan • M. J. Lawrence • B. O'Connell
}

Published online: 1 June 2007

(C) Springer-Verlag 2007

\begin{abstract}
Rapid accurate detection is a prerequisite for the successful control of meticillin-resistant Staphylococcus aureus (MRSA). The IDI-MRSA real-time polymerase chain reaction (PCR) assay was designed to provide rapid results from nasal specimens collected in Stuart's liquid transport medium. This study has evaluated the IDI-MRSA kit for use in a clinical laboratory by investigating the following parameters: (1) limits of detection (LoD), (2) performance with Amies' gel-based transport medium, (3) ability to detect strains of MRSA in a collection represen-
\end{abstract}

\footnotetext{
A. S. Rossney $(\bowtie) \cdot$ M. M. Fitzgibbon $\cdot$ P. M. Morgan $\cdot$

B. O'Connell

National MRSA Reference Laboratory, St. James's Hospital,

James's Street,

Dublin 8, Ireland

e-mail: arossney@stjames.ie

C. M. Herra

Department of Microbiology St. James's Hospital and Department of Clinical Microbiology, University of Dublin, Trinity College, James's Street,

Dublin 8, Ireland
}

A. S. Rossney • M. M. Fitzgibbon • B. O'Connell

Department of Clinical Microbiology, University of Dublin

Trinity College St. James's Hospital,

James's Street,

Dublin 8 , Ireland

\section{J. Lawrence · B. O’Connell}

Department of Microbiology, St. James's Hospital,

James's Street,

Dublin 8, Ireland

Present address:

C. M. Herra

School of Biological Sciences, Dublin Institute of Technology, Kevin Street,

Dublin 8, Ireland tative of MRSA in Ireland since $1974(n=113)$ and (4) performance in a clinical trial with swabs from nose, throat and groin/perineum sites from 202 patients. LoDs (colony-forming units per $\mathrm{ml}$ ) of the IDI-MRSA kit, direct culture on MRSA-Select chromogenic agar (CA) and saltenrichment culture (with subculture onto CA) were $10^{3}$, $10^{3}$ and $10^{2}$, respectively. LoDs with Stuart's and Amies' transport media were comparable. All except one of the 113 MRSA isolates were detected by the kit but, when six control strains carrying staphylococcal cassette chromosome mec (SCCmec) type IV element subtypes IVa-d and SCCmec types $\mathrm{V}$ and $\mathrm{V}_{\mathrm{T}}$ were tested, the kit failed to detect MRSA carrying SCCmec V. The sensitivity and specificity for detection of MRSA from nose, throat and groin/ perineum specimens were comparable with slightly lower sensitivities from throat and groin/perineum specimens compared with nasal swabs $(90 \%, 97 \% ; 89 \%, 99 \% ; 88 \%$, $99 \%$, respectively). Overall sensitivity, specificity and positive and negative predictive values for specimens from all sites were $88 \%, 99 \%, 94 \%$ and $97 \%$, respectively. Further developments to improve the sensitivity of this highly worthwhile assay are required.

\section{Introduction}

Rapid detection of meticillin-resistant Staphylococcus aureus (MRSA) is important for the early implementation of effective infection control [1]. Meticillin resistance is encoded by mecA, which is carried on the staphylococcal cassette chromosome mec ( $\mathrm{SCCmec}$ ). Previous molecular methods for the rapid detection of MRSA have investigated the presence of mecA and nuc or fem but have been unable to distinguish between MRSA and mixtures of meticillinsusceptible Staphylococcus aureus (MSSA) and meticillin- 
resistant coagulase-negative staphylococci (MR-CNS) [1]. The IDI-MRSA kit (GeneOhm Sciences Canada, Ste-Foy, QC, Canada) overcomes this problem with a real-time polymerase chain reaction (PCR) assay designed to detect MRSA-specific DNA sequences within SCCmec, although one study has shown that this method failed to detect 21 MRSA isolates and incorrectly identified 26 MSSA as MRSA [2]. Newer versions of the kit (the most recent in March 2006) incorporate modified primer and probe design to improve efficacy but to date, there are no published reports of the value of these modifications.

The IDI-MRSA kit is marketed as a screening method for the detection of MRSA from nasal swabs from patients at risk for colonisation with MRSA and has a quoted limit of detection (LoD) of 325 colony-forming units (CFU) per swab [3]. However, screening protocols relying on the culture of nasal specimens alone may detect only $79 \%$ of MRSA carriers, whereas culture of specimens from the nose, throat and groin/perineum will detect up to $98 \%$ of carriers [4]. In addition, the IDI-MRSA kit is validated for use with Stuart's liquid transport medium but many institutions use transport swabs containing Amies' gel-based transport medium. The present study has been designed to evaluate the IDI-MRSA kit for use in a clinical laboratory. The following parameters have been investigated: (1) LoD, (2) performance with Amies' gel-based transport medium, (3) ability to detect strains of MRSA in a collection representative of MRSA in Ireland since 1974 and (4) performance in a clinical trial with swabs from throat and groin/perineum sites in addition to nasal specimens.

\section{Materials and methods}

\section{LoD assays}

LoD assays were performed on four MRSA isolates that exhibited a range of oxacillin minimum inhibitory concentrations (MIC) and were representative of strains currently or recently prevalent in Ireland $[5,6]$. These isolates comprised: (1) ST22-MRSA-IV, MIC $>256 \mathrm{mg} / \mathrm{l}$; (2) ST22-MRSA-IV, MIC $32 \mathrm{mg} / \mathrm{l}$; (3) ST8-MRSA-IID, MIC >256 mg/l; (4) an isolate with an MIC of $4.0 \mathrm{mg} / 1$ that was $m e c A$-positive by PCR and expressed PBP2a but for which multilocus sequence typing (MLST) data and SCCmec-typing results were unavailable.

LoD assays were performed by preparing saline suspensions of each isolate from overnight cultures grown on Columbia agar (LabM Lab1; International Diagnostics Group, Bury, UK) containing 7\% (w/v) horse blood (blood agar; BA) to a density equivalent to a 0.5 McFarland turbidity standard. Following an initial 1/100 dilution, suspensions were diluted in a series of 10 -fold dilutions (from $10^{5}$ to $\left.10^{1}\right)$. Colony counts $(\mathrm{CFU} / \mathrm{ml})$ for each dilution were determined by spiral plating onto brain heart infusion (BHI) agar (Oxoid CM375; Oxoid, Basingstoke, UK) using a WASP spiral plater (Don Whitley Scientific, Shipley, UK). Each dilution was adsorbed onto two Copan Stuart's liquid transport medium swabs (Copan 141C; Copan Italia, Brescia, Italy) and used to determine the LoD of each isolate in pure culture.

LoDs for all four MRSA isolates were also determined in the presence of a "cocktail" of bacteria consisting of 10 MSSA isolates (which were mecA-negative by in-house conventional PCR), five MR-CNS and single isolates of Moraxella catarrhalis, Escherichia coli and Candida species. All MSSA isolates were grown overnight on BA, suspended in saline to a density equivalent to a 0.5 McFarland standard, pooled, diluted to approximately $10^{6} \mathrm{CFU} / \mathrm{ml}$, adsorbed onto a Copan transport swab and tested with the IDI-MRSA kit (GeneOhm Sciences Canada). The CNS and remaining bacterial species were prepared and tested similarly. All isolates were combined to form a "cocktail" to mimic normal flora and re-tested with the IDIMRSA kit to ensure that no false-positive results were obtained. Ten-fold dilutions (from $10^{5}$ to $10^{1}$ ) of the four test isolates were prepared by using the "cocktail" (at a set concentration of $10^{5} \mathrm{CFU} / \mathrm{ml}$ ) as diluent and adsorbed onto two Copan Stuart's liquid transport medium swabs.

One Copan swab from each preparation of the four MRSA isolates in pure culture and in the presence of mixed flora was investigated for the presence of MRSA by using the IDI-MRSA kit on a Smart Cycler II thermal cycler (Cepheid, Sunnyvale, Calif., USA). The kit assay was performed according to the manufacturer's instructions with two modifications. After the swabs were vortexed in sample buffer, the tubes were centrifuged at $1,000 \mathrm{x} g$ for $30 \mathrm{~s}$ to minimize aerosol formation. To minimize the risk of contaminating the negative control included in the kit, aliquots sufficient for single use were dispensed into individual microcentrifuge tubes. In all cases, real-time PCR was performed immediately after DNA extraction.

Swabs tested with the IDI-MRSA kit were cultured by salt enrichment by adding $1 \mathrm{ml}$ tryptic soy broth (TSB; BD 211825; Becton Dickinson and Company, Sparks, Md, USA) containing $6.5 \% \mathrm{NaCl}$ (salt TSB) to the residual swabs in the sample buffer tubes after the sample buffer had been removed. After $18 \mathrm{~h}$ incubation at $35^{\circ} \mathrm{C}$, enrichment broths $(10-\mu \mathrm{l}$ volumes) were subcultured onto BA, MRSA-Select chromogenic agar (CA; Bio-Rad 63747; Bio-Rad Life Science Group, Marnes La Coquette, France) and mannitol salt agar (BD 11407; Becton Dickinson) containing $5 \mathrm{mg} / 1$ meticillin (Sigma M6535, Sigma-Aldrich, Poole, England) (MMSA).

The second swab of each of the MRSA preparations was cultured onto BA, CA and MMSA. All isolates from enriched and direct culture were identified as Staphyloco- 
ccus aureus by detecting staphylocoagulase production (tube coagulase test) and, if necessary, clumping factor (Pastorex Staph Plus; Bio-Rad). Meticillin resistance was tested by disk diffusion at $30^{\circ} \mathrm{C}$ by using $10-\mu \mathrm{g}$ meticillin disks (Oxoid) on BA and with $30-\mu \mathrm{g}$ cefoxitin disks on Mueller-Hinton agar (BD 211438; Becton Dickinson) according to Clinical and Laboratory Standards Institute (CLSI) guidelines [7, 8]. LoDs for both culture methods were calculated on the basis of growth of $>1$ colony on a single subculture medium or $\geq 1$ colony on more than one subculture medium and were expressed as the average of the counts obtained with the four isolates.

Evaluation of Transwabs with Amies' clear transport medium

Transwabs with Amies' clear transport medium [MW170; Medical Wire and Equipment (MWE), Corsham, England] were evaluated by repeating the $\mathrm{LoD}$ determination of the four test isolates in pure culture and in the presence of the mixed flora "cocktail". Test isolate suspensions were adsorbed onto Transwabs, investigated by using the IDIMRSA kit and cultured as described above.

\section{MRSA strains}

In total, 119 isolates were investigated. These included 113 MRSA isolates representative of MRSA recovered in Ireland and six control strains. The former comprised 89 isolates representative of most epidemiological types recovered between 1974 and 2003, six isolates that were not investigated by MLST and SCCmec typing and 18 Panton-Valentine leucocidin (PVL)-positive community-acquired MRSA [5, 6]. Details of these isolates are summarised in Table 1. The six MRSA control strains represented isolates carrying SCCmec IV subtypes IVa, IVb, IVc and IVd and SCCmec $\mathrm{V}$ and $\mathrm{V}_{\mathrm{T}}$ because there were no data on MRSA carrying these SCCmec elements in Ireland $[9,10]$.

Isolates were prepared at concentrations of $\sim 10^{2} \mathrm{CFU} / \mathrm{ml}$ above the LoD of the kit (as determined in the present study) in saline suspensions containing $\sim 10^{5} \mathrm{CFU} / \mathrm{ml}$ of the mixed flora "cocktail" (described above), adsorbed onto MWE Transwabs and tested by using the IDI-MRSA kit according to the manufacturer's instructions. After being tested, all swabs were cultured by salt enrichment and subcultured onto BA, CA and MMSA as described above.

Any isolate that was negative with the kit was re-tested in pure culture and at one 10-fold higher concentration; if still negative, the inoculum was further increased to $\sim 10^{8} \mathrm{CFU} / \mathrm{ml}$. The mecA status was confirmed by a conventional in-house end-point PCR assay [11]. The DNA extracted for conventional PCR was also tested by using the IDI-MRSA kit. DNA extracted with the IDI-
Table 1 Meticillin-resistant Staphylococcus aureus (MRSA) isolates used to determine whether the IDI-MRSA kit could detect all strains of MRSA prevalent in Ireland ( $n=113 ; N D$ not done, NT AR patterns designated "no type" pending results of DNA macrorestriction digestion analysis, PVL Panton-Valentine leucocidin, SCV small colony variant, $v$ previously reported variants of SCCmec $[6,20]$ )

\begin{tabular}{lll}
\hline $\begin{array}{l}\text { Multilocus } \\
\text { sequence type }\end{array}$ & SCCmec & Antibiogram-resistogram (AR) type \\
\hline ST5 $^{\text {a }}(n=10)$ & II & $07.3^{\text {a }}(n=3) ; 07.4(n=5) ; 11(n=2)$ \\
ST5 $(n=1)$ & IV & Unfamiliar $^{\text {b }}(n=1)$ \\
ST8 $^{\text {c }(n=11)}$ & IV or & $43(n=8) ;$ unfamiliar $^{\text {b }}\left(n=3^{\mathrm{c}}\right)$ \\
& IVv & \\
ST8 $(n=26)$ & II or IIv & $13(n=8) ; 14(n=15) ;$ New03 $(n=3)$ \\
ST12 $(n=1)$ & IV & NT $(n=1)$ \\
ST22 $(n=14)$ & IV & $06(n=13) ;$ NT $(n=1)$ \\
ST30 $(n=2)$ & IV & NT $(n=2)$ \\
ST36 $(n=3)$ & II & $07.0(n=2) ; 07.2(n=1)$ \\
ST45 $(n=1)$ & IV & NT $(n=1)$ \\
ST239 $(n=13)$ & III or & $01(n=4) ; 09(n=3) ; 15(n=2) ; 44$ \\
& IIIv & $(n=3) ; 23(n=1)$ \\
ST247 $(n=3)$ & IA & $22(n=2) ;$ New02 $(n=1)$ \\
ST250 $(n=4)$ & I or Iv & $02(n=4)$ \\
ND $(n=6)$ & ND & $07(n=2) ;$ New02 $(n=1) ; 06(n=1) ;$ \\
& & SCV $(n=2)$ \\
ND $(n=18)$ & ND & PVL-positive community-acquired \\
& & MRSA $(n=18)$
\end{tabular}

${ }^{\text {a }}$ One isolate was a double locus variant of ST5

${ }^{\mathrm{b}}$ Unfamiliar AR pattern

${ }^{\mathrm{c}}$ Two isolates were single locus variants of ST8

MRSA kit from isolates still yielding negative results was also tested by conventional PCR. DNA extracted with the IDI-MRSA kit from Staphylococcus aureus ATCC 43300 was included as a positive control with this PCR assay.

\section{Clinical trial}

Clinical specimens were obtained from patients attending St. James's Hospital, a large 936-bed tertiary-referral adult university teaching hospital. Specimens from nose, throat and groin/perineum collected for routine MRSA screening in the clinical microbiology laboratory were tested. Criteria for routine screening were admission to a critical care area, admission from another hospital or long-stay care institution, previous MRSA, recent hospitalisation in a foreign hospital and pre- and post-operative cardiac surgery. Specimens from 202 consecutive patients in whom all three sites were sampled were included in the study. Duplicate specimens from the same patient were excluded. Specimens were tested with the IDI-MRSA kit after they had been subjected to routine culture by direct inoculation onto $\mathrm{CA}$ in the diagnostic laboratory. Specimens were tested within $24 \mathrm{~h}$ of collection from the diagnostic laboratory and stored at $4^{\circ} \mathrm{C}$ until investigations were complete. 
After being tested with the IDI-MRSA kit, all specimens were cultured by salt enrichment as described above and subcultured onto BA, CA and MMSA. Suspect colonies were identified as MRSA as described above and the presence of mecA was confirmed by a conventional inhouse end-point PCR assay [11]. MRSA isolates recovered from specimens yielding kit-negative results were prepared in suspensions at concentrations of $10^{5} \mathrm{CFU} / \mathrm{ml}$ in the presence of the mixed flora "cocktail", adsorbed onto Transwabs and re-tested by using the IDI-MRSA kit. Bacterial growth from BA plates from specimens that tested IDI-MRSA kit-positive but from which MRSA was not recovered in culture was similarly tested to exclude the possibility that such positive results occurred as a result of MSSA or MR-CNS.

\section{Control strains}

Staphylococcus aureus ATCC 25923 and Staphylococcus aureus ATCC 43300 were used as negative and positive controls, respectively. Control isolates were prepared in saline suspensions containing $10^{5} \mathrm{CFU} / \mathrm{ml}$, adsorbed onto appropriate swabs and processed with each batch of tests. Positive and negative controls were included with each batch of tests when determining LoDs. A negative control was included with each batch of MRSA strains tested. A positive control was included with every batch of specimens in the clinical trial. Staphylococcus aureus ATCC 43300 was included with each batch of CA and MMSA plates used.

\section{Statistics}

In the clinical trial, the sensitivity, specificity and positive and negative predictive values (with $95 \%$ confidence intervals) of the IDI MRSA kit were calculated for each specimen type compared with direct culture, enrichment culture and the combined results from both direct and enrichment culture methods. The sensitivity, specificity and positive and negative predictive values of the kit were also calculated from results obtained from specimens from all sites.

Because the IDI-MRSA kit detects DNA that may come from non-viable MRSA, whereas culture detects viable organisms only, IDI-MRSA kit-positive specimens that were culture-negative were considered as possible "true"positive results if the patient had been previously positive for MRSA or if the patient was positive at another site. Obversely, with salt enrichment culture, specimens from patients with no record of being previously positive and that yielded one or two colonies only on one of the two selective media used were considered possible "false"positive results. Sensitivity, specificity and positive and negative predictive values were also calculated by using these "amended" results. Further calculations were made when patients were grouped into those who were known to have been previously positive for MRSA and those who were not known to have been previously positive.

\section{Results}

LoD assays

LoDs of the IDI-MRSA kit, direct culture and salt enrichment culture for the four test isolates prepared in pure culture by using Stuart's and Amies' transport medium swabs are shown in Table 2. The LoD of the IDI-MRSA kit was $10^{3} \mathrm{CFU} / \mathrm{ml}$ for both Stuart's liquid transport medium and Amies' gel-based transport medium. This LoD was comparable to the value obtained for direct culture but was 10-fold higher than the LoD of enrichment culture with either transport medium. There was no appreciable difference in $\mathrm{LoD}$ values for isolates prepared in pure culture or mixed flora, with both types of transport medium.

\section{MRSA strains}

Isolate suspensions were prepared in saline at a concentration of $\sim 10^{5} \mathrm{CFU} / \mathrm{ml}$ (i.e. $\sim 10^{2} \mathrm{CFU} / \mathrm{ml}$ above the $\mathrm{LoD}$ determined in the present study). All isolates except two yielded positive IDI-MRSA kit results. Both negative isolates were mecA-positive by conventional PCR. One isolate was a hetero-glycopeptide-intermediate Staphylococcus aureus (hGISA) which was kit-positive when retested in pure culture from a suspension at a concentration of $10^{5} \mathrm{CFU} / \mathrm{ml}$. Five additional hGISA isolates (prepared from suspensions at $10^{5} \mathrm{CFU} / \mathrm{ml}$ ) were tested with the kit and all were positive. The second isolate that failed to yield a positive IDI-MRSA kit result was a Staphylococcus aureus control strain carrying the SCCmec V element. This isolate remained negative with the IDI-MRSA kit when tested in pure culture (at concentrations prepared from

Table 2 Limits of detection $\{L o D$; average bacterial counts in colony-forming units $(\mathrm{CFU} / \mathrm{ml})\}$ for the IDI-MRSA kit, direct culture and salt enrichment broth culture with either Stuart's or Amies' transport medium

\begin{tabular}{lll}
\hline Method & LoD & \\
\cline { 2 - 3 } & $\begin{array}{l}\text { Stuart's medium } \\
(\mathrm{CFU} / \mathrm{ml})^{\mathrm{a}}\end{array}$ & $\begin{array}{l}\text { Amies' medium } \\
(\mathrm{CFU} / \mathrm{ml})^{\mathrm{a}}\end{array}$ \\
\hline IDI-MRSA & $2.0 \times 10^{3}$ & $1.0 \times 10^{3}$ \\
Direct culture & $1.3 \times 10^{3}$ & $0.8 \times 10^{3}$ \\
Enrichment culture & $1.4 \times 10^{2}$ & $2.4 \times 10^{2}$ \\
\hline
\end{tabular}

${ }^{\text {a }}$ Bacterial counts (in $\mathrm{CFU} / \mathrm{ml}$ ) of saline suspensions adsorbed onto relevant transport medium swabs prior to testing the swabs by each method 
suspensions of $10^{5}, 10^{6}$ and $10^{8} \mathrm{CFU} / \mathrm{ml}$ ) but DNA extracted for conventional PCR yielded a positive result when tested with the IDI-MRSA kit. Conversely, DNA extracted with the IDI-MRSA kit was negative when tested by conventional PCR but DNA extracted with the IDIMRSA kit from Staphylococcus aureus ATCC 43300 was also negative by conventional methods. All isolates were successfully recovered from suspensions containing mixed flora when cultured by salt enrichment.

\section{Clinical trial}

A total of 606 specimens from 202 patients was investigated. An overview of the numbers of positive specimens by the IDI-MRSA kit, by direct and enrichment culture and by "amended" results is shown in Table 3. MRSA was detected in 120 specimens $(20 \%, 120 / 606)$ from 63 patients $(31 \%, 63 / 202)$ by the IDI-MRSA kit, with $93(15 \%)$ and $116(19 \%)$ specimens being positive by direct and enrichment culture, respectively. When the results of both direct and enrichment culture methods were combined, 119 specimens from 61 patients were positive $(20 \%, 119 / 606$; $30 \%, 61 / 202)$. Of the 119 culture-positive specimens, 98 were positive by the IDI-MRSA kit. Twelve specimens from 11 patients were unresolved on initial testing but, after repeat testing following freezing at $-20^{\circ} \mathrm{C}$, only three specimens remained unresolved.

Twenty-two culture-negative specimens were positive by the IDI-MRSA kit but only 15 of these were deemed to be

Table 3 Overview of results obtained with the IDI-MRSA kit and culture from 606 specimens

\begin{tabular}{lllll}
\hline \multirow{2}{*}{ Culture method } & & \multicolumn{2}{l}{ IDI-MRSA kit } \\
\cline { 3 - 5 } & & Positive & Negative & Total \\
\hline Direct culture & Positive & 85 & 8 & 93 \\
Enrichment culture & Negative & 35 & 475 & 510 \\
& Positive & 97 & 19 & 116 \\
Direct and enrichment & Negative & 23 & 464 & 487 \\
& Positive & 98 & $21^{\mathrm{a}}$ & 119 \\
Direct and enrichment, & Negative & 22 & 462 & 484 \\
amended results & Positive & 113 & 15 & 128 \\
\multirow{2}{*}{ Total } & & & & \\
\hline
\end{tabular}

${ }^{a}$ Of 26 specimens positive by salt enrichment only, five were deemed false-positive because only one or two colonies grew from either CA or MMSA and the patients had no previous MRSA and no other screening sites that were positive

${ }^{b}$ Amended results included numbers of specimens that were culturepositive and those that were positive by the IDI-MRSA kit but culture-negative from previously positive patients or from patients from whom a specimen from another site was positive

${ }^{\mathrm{c}}$ Three isolates were unresolved by the IDI-MRSA kit true positives because the patients had a previous MRSA isolate or were positive at another site. Twenty-six specimens were positive by salt enrichment culture only but five of these were deemed false-positive because only one or two colonies grew from either CA or MMSA and the patients had no previous MRSA and no other screening sites that were positive. When calculations were performed based on these "amended" results, 128 specimens $(21 \%$; 128/606) from 64 patients $(32 \%$; 64/202) were deemed positive (Table 3).

When representative MRSA isolates recovered from six of the 15 specimens that were IDI-MRSA kit-negative and MRSA culture-positive were further investigated, all isolates tested positive with the kit. Non-MRSA isolates recovered from specimens that were kit-positive and MRSA culture-negative were also tested by the kit but all isolates yielded negative results with the IDI-MRSA kit.

\section{Statistics}

The sensitivity, specificity and positive and negative predictive values (with $95 \%$ confidence intervals) of the IDI-MRSA kit compared with direct culture, enrichment culture and amended results are shown in Table 4. With nasal swabs, the improved yield of MRSA following salt enrichment resulted in a decrease in the comparative sensitivity of the kit from $89 \%$ to $81 \%$ but when the "amended" results were considered, the sensitivity of the kit compared with culture (by both direct and enrichment methods) was $90 \%$. Amended results obtained from nose, throat and groin/perineum specimens were comparable, although sensitivity decreased slightly with throat and groin/perineum specimens (from $90 \%$ to $89 \%$ and $88 \%$ ).

When the IDI-MRSA kit results from specimens from all sites were compared with "amended" culture results, the overall sensitivity, specificity and positive and negative predictive values were $88 \%, 99 \%, 94 \%$ and $97 \%$, respectively. When this analysis was restricted to specimens from previously positive patients, the sensitivity was $92 \%$ but dropped to $82 \%$ with specimens from patients with no record of being previously positive. For the latter group, the negative predictive value was $98 \%$ but these data should be interpreted with caution because of the lower positivity rate of $9 \%$ compared with the $39 \%$ positivity rate in specimens from previously positive patients.

\section{Discussion}

The clinical need for the rapid detection of MRSA to implement effective infection control measures requires that the methods be both sensitive and specific. The inherent sensitivity, specificity and speed of real-time PCR make 
Table 4 Sensitivity, specificity and positive $(P P V)$ and negative $(N P V)$ predictive values expressed as a percentage for the IDI-MRSA kit compared with culture from nose, throat and groin/perineum specimens (numbers in parentheses 95\% confidence intervals, All, $P P P$ all sites from previously positive patients, All, NPP all sites from patients with no record of being previously positive)

\begin{tabular}{|c|c|c|c|c|c|}
\hline \multirow[t]{2}{*}{ Culture mthod } & \multirow[t]{2}{*}{ Site } & \multicolumn{4}{|c|}{ IDI-MRSA kit } \\
\hline & & Sensitivity & Specificity & PPV & NPV \\
\hline Direct & Nose & 89 (78-99) & 89 (85-94) & $63(50-77)$ & $97(95-99.9)$ \\
\hline Enrichment & Nose & $81(70-93)$ & $91(87-96)$ & $71(59-84)$ & $95(91-98)$ \\
\hline Both methods ${ }^{\mathrm{a}}$ & Nose & $81(70-93)$ & $91(87-96)$ & $71(59-84)$ & $95(91-98)$ \\
\hline Amended $^{\mathrm{a}, \mathrm{b}}$ & Nose & $90(82-98)$ & $97(95-99.9)$ & $92(84-99.6)$ & $97(94-99.5)$ \\
\hline Amended $^{\mathrm{a}, \mathrm{b}}$ & Throat & 89 (82-99) & $99(98-100)$ & $98(93-100)$ & $97(94-99.5)$ \\
\hline Amended $^{\mathrm{a}, \mathrm{b}}$ & Groin/perineum & $88(76-99)$ & $99(97-100)$ & $93(84-100)$ & $98(95-99.9)$ \\
\hline Both methods ${ }^{\mathrm{a}}$ & All sites & $82(76-89)$ & 96 (94-97) & $82(75-89)$ & $96(94-98)$ \\
\hline Amended $^{\mathrm{a}, \mathrm{b}}$ & All sites & $88(83-94)$ & $99(97-99.6)$ & $94(90-98)$ & $97(95-98)$ \\
\hline Both methods ${ }^{\mathrm{a}}$ & All, PPP & $88(80-94)$ & $92(87-96)$ & $85(77-91)$ & 93 (89-97) \\
\hline Amended $^{\mathrm{a}, \mathrm{b}}$ & All, PPP & $92(86-97)$ & $99(97-100)$ & $98(95-100)$ & $95(92-98)$ \\
\hline Both methods ${ }^{\mathrm{a}}$ & All, NPP & $68(51-84)$ & $98(96-99)$ & $72(56-89)$ & 97 (95-99) \\
\hline Amended $\mathrm{d}^{\mathrm{a}, \mathrm{b}}$ & All, NPP & $82(68-96)$ & $98(97-99.6)$ & $79(65-94)$ & $98(97-99.8$ \\
\hline
\end{tabular}

${ }^{a}$ Direct and enrichment culture results

${ }^{\mathrm{b}}$ Amended results: see footnote to Table 3

this technique an ideal candidate for the swift identification of MRSA [12]. The IDI-MRSA kit is the first commercial molecular assay for the rapid detection of MRSA that can distinguish specimens containing MRSA from those containing mixtures of MSSA and MR-CNS [2].

The manufacturer's instructions for the use of the IDIMRSA kit state that the kit has a LoD of 325 CFU per swab [3], which is disappointingly high for a molecular detection assay. However, the present study has found that the kit has an even lower sensitivity, with the LoD being 10 -fold higher than the quoted value and no better than the LoD of direct culture on CA. Although the CA used in the present study (MRSA-Select) has recently been shown to recover MRSA with a sensitivity of $97 \%-99 \%$ compared with other culture media [13, 14], a molecular assay would be expected to have a lower LoD than culture [12]. However, our study has shown that salt enrichment culture followed by subculture onto CA and MMSA yields an improved sensitivity and a 10-fold lower LoD compared with the kit. The poor sensitivity demonstrated by the kit may occur because of the small volume of template DNA $(2.8 \mu \mathrm{l})$ used in the kits, the complexity of the multiplex reactions, the low assay volume utilised by the PCR and/or the relatively crude DNA extraction protocol used.

In addition to assessing the sensitivity of the kit, the present study aimed to evaluate whether Transwabs containing Amies' gel-based transport medium could be used with the IDI-MRSA kit. Prior to testing, the possibility that the gel consistency of Amies' transport medium compared with the liquid in Stuart's transport medium might adversely effect DNA extraction was considered but the results obtained with the LoD assays showed that the use of Amies' transport medium did not result in any loss of sensitivity.

Another question addressed was whether the kit could detect all strains of MRSA prevalent in Ireland. With the exception of one hGISA isolate, all isolates were detected. The hGISA isolate was detected on repeat testing in pure culture suggesting that the problem lay in the DNA extraction protocol and not with detection of the SCCmec element carried by the isolate. No difficulty was experienced in obtaining positive results when an additional five hGISA isolates were tested with the IDI-MRSA kit. The kit also yielded a negative result with the control isolate carrying SCCmec type $\mathrm{V}$ but a kit-positive result was obtained from DNA extracted for conventional PCR. The failure of the kit to detect these isolates suggests that the kit may have difficulty extracting DNA from some isolates. Addition of lysostaphin, prolonged or more vigorous mixing or the use of another DNA extraction method are aspects that remain to be investigated but it is worrying that SCCmec V, which is one of the two SCCmec elements associated with community-acquired MRSA, was not detected.

Although the present study has shown that throat and groin/perineum specimens are suitable for use with the IDIMRSA kit, the overall sensitivity of the kit in the clinical trial was $88 \%$, which is disappointingly low. Previous evaluations of the IDI-MRSA kit quoted sensitivity values of $92 \%-100 \%$ but comparisons were made against direct culture on MSA and, when enrichment culture was used, subcultures were made onto BA [15, 16]. More recently, sensitivities of $89 \%$ with nasal specimens and $82 \%$ with groin specimens were reported when comparison was made 
against direct and enrichment culture on MSA containing oxacillin (OMSA) [17]. Studies comparing culture media selective for MRSA have shown that OMSA has sensitivities ranging from $60 \%$ to $84 \%$, whereas the CA used in the present study (MRSA-Select) has a sensitivity of $97 \%-99 \%$ $[1,13,14]$. Another evaluation of the IDI-MRSA kit reported a sensitivity of $100 \%$ for the kit compared with culture on CHROMagar MRSA but, again, that medium was found to have a sensitivity of only $83 \%[13,18]$. In the present study, the inclusion of salt enrichment culture increased the number of MRSA-positive specimens by $23 \%(21 / 93)$ and, as a result, the relative sensitivity of the kit for nasal specimens decreased from $89 \%$ when compared with direct culture, to $81 \%$ when compared with salt enrichment culture. With a LoD of $10^{3} \mathrm{CFU} / \mathrm{ml}$ and a sensitivity of $82 \%$ for specimens from all sites among patients who had no record of being previously positive for MRSA, the IDI-MRSA kit is not a replacement for culture.

Whereas it was important to determine that both throat and groin/perineum sites were suitable for use with the kit, the cost of testing all three sites would be prohibitively expensive (the kit price per test is approximately €25). Although costs can be reduced by pooling specimens from various sites in a selective broth and, following overnight incubation, by using the kit to detect MRSA in the broth culture, this method has been associated with a high rate of false-positive results [19].

In the present study, when non-MRSA bacterial isolates recovered on BA from MRSA-culture-negative kit-positive specimens were investigated further, no isolates tested kitpositive. It is unlikely that the kit-positive results occurred because the kit was more sensitive than culture (because salt enrichment culture was shown to be the more sensitive detection method) but positive results may have occurred because the kit detected DNA that may have derived from non-viable organisms. Important questions in this regard are how long does DNA remain detectable after successful MRSA eradication and what should be the clinical interpretation of such results. The possibility that a patient from whom MRSA has been successfully eradicated might subsequently re-acquire the organism is a further complication. Among MRSA isolates recovered from kit-negative culture-positive specimens, all isolates yielded kit-positive results when re-tested in saline suspensions at concentrations of $10^{5} \mathrm{CFU} / \mathrm{ml}$. This finding suggests that the falsenegative results do not arise from an inability to detect a particular SCCmec type but again may reflect the low sensitivity of the kit or inadequate DNA extraction.

Although the kit produces rapid results relative to enrichment culture, the overall assay time including sample preparation takes approximately $2.5 \mathrm{~h}$ for batches of 12 specimens. It also has the disadvantage that results are displayed only as positive, negative or unresolved and, hence, information from amplification plots cannot be accessed to aid in the interpretation of anomalous results.

Providing an overall evaluation of the kit is complicated by the fact that the kit detects DNA, whereas culture detects viable organisms. A major difficulty is defining "true"positive and -negative specimens. In the present study, data were analysed by direct comparison with culture but, to overcome the problem of considering all kit-positive culture-negative specimens as false-positive results, kit performance was also analysed by using "amended" results where kit-positive culture-negative results from patients who were previously positive for MRSA were regarded as "true" positive results. Other studies have excluded specimens from patients on anti-staphylococcal therapy and only included patients fulfilling stringent criteria of high-risk for MRSA acquisition $[15,16]$. In the present study, no attempt was made to pre-select patients on the basis of antibiotic therapy or risk factors other than those considered for routine MRSA screening in this institution. Although the manufacturer specifies that the kit's intended use is with specimens from patients at high risk for MRSA, in clinical practice, the need for rapid results may be equally pressing in other groups of patients (for example, pre-operative cardiac surgery patients). However, when data were analysed from patients with no previous record of MRSA, the sensitivity was $82 \%$. The kit did, however, show the highest negative predictive value with specimens from this group of patients but the low prevalence of MRSA in this group must be considered when interpreting results. In conclusion, the kit can provide a rapid preliminary screen for MRSA but the final result requires culture. Further developments to improve the sensitivity of this highly worthwhile assay are required.

Acknowledgements We thank Teruyo Ito (Juntendo University, Tokyo, Japan) and Robert Daum (University of Chicago, Chicago, Ill., USA) for the gift of SCCmec IV and SCCmec V control strains. We are grateful to BioFact (Ireland) for providing the SmartCycler platform and for supplying IDI-MRSA kits at an evaluation rate. The experiments reported in this article comply with current laws in Ireland.

\section{References}

1. Brown DF, Edwards DI, Hawkey PM, Morrison D, Ridgway GL, Towner KJ, Wren MW (2005) Guidelines for the laboratory diagnosis and susceptibility testing of methicillin-resistant Staphylococcus aureus (MRSA). J Antimicrob Chemother 56:10001018

2. Huletsky A, Giroux R, Rossbach V, Gagnon M, Vaillancourt M, Bernier M, Gagnon F, Truchon K, Bastien M, Picard FJ, Belkum A van, Ouellette M, Roy PH, Bergeron MG (2004) New real-time PCR assay for rapid detection of methicillin-resistant Staphylococcus aureus directly from specimens containing a mixture of staphylococci. J Clin Microbiol 42:1875-1884 
3. Anon (2006) IDI-MRSA Assay Package Insert. http://www. geneohm.com/english/idi_mrsa.htm. Cited 14 February 2007

4. Anon (1998) Revised guidelines for the control of methicillinresistant Staphylococcus aureus infection in hospitals. British Society for Antimicrobial Chemotherapy, Hospital Infection Society and the Infection Control Nurses' Association. J Hospital Infect 39:253-290

5. Rossney AS, Lawrence MJ, Morgan PM, Fitzgibbon MM, Shore A, Coleman DC, Keane CT, O'Connell B (2006) Epidemiological typing of MRSA isolates from blood cultures taken in Irish hospitals participating in the European Antimicrobial Resistance Surveillance System (1999-2003). Eur J Clin Microbiol Infect Dis 25:79-89

6. Shore A, Rossney AS, Keane CT, Enright MC, Coleman DC (2005) Seven novel variants of the staphylococcal chromosomal cassette mec in methicillin-resistant Staphylococcus aureus isolates from Ireland. Antimicrob Agents Chemother 49:2070-2083

7. Hewitt JH, Coe AW, Parker MT (1969) The detection of methicillin resistance in Staphylococcus aureus. J Med Microbiol 2:443-456

8. Clinical and Laboratory Standards Institute (2006) Performance Standards for Antimicrobial Susceptibility Testing; Sixteenth Informational Supplement. CLSI Document M100-S16. Clinical and Laboratory Standards Institute, 940 West Valley Road, Suite 1400, Wayne, Pennsylvania 19087-1898, USA

9. Boyle-Vavra S, Ereshefsky B, Wang CC, Daum RS (2005) Successful multiresistant community-associated methicillin-resistant Staphylococcus aureus lineage from Taipei, Taiwan, that carries either the novel staphylococcal chromosome cassette mec (SCCmec) type $\mathrm{V}_{\mathrm{T}}$ or SCCmec type IV. J Clin Microbiol 43:4719-4730

10. Chongtrakool P, Ito T, Ma XX, Kondo Y, Trakulsomboon S, Tiensasitorn C, Jamklang M, Chavalit T, Song JH, Hiramatsu K (2006) Staphylococcal cassette chromosome mec (SCCmec) typing of methicillin-resistant Staphylococcus aureus strains isolated in 11 Asian countries: a proposal for a new nomenclature for SCCmec elements. Antimicrob Agents Chemother 50:1001-1012

11. Oliveira DC, Lencastre H de (2002) Multiplex PCR strategy for rapid identification of structural types and variants of the mec element in methicillin-resistant Staphylococcus aureus. Antimicrob Agents Chemother 46:2155-2161
12. Cockerill FR 3rd, Smith TF (2004) Response of the clinical microbiology laboratory to emerging (new) and reemerging infectious diseases. J Clin Microbiol 42:2359-2365

13. Nsira SB, Dupuis M, Leclercq R (2006) Evaluation of MRSA Select, a new chromogenic medium for the detection of nasal carriage of methicillin-resistant Staphylococcus aureus. Int J Antimicrob Agents 27:561-564

14. Stoakes L, Reyes R, Daniel J, Lennox G, John MA, Lannigan R, Hussain Z (2006) Prospective comparison of a new chromogenic medium, MRSASelect, to CHROMagar MRSA and mannitol-salt medium supplemented with oxacillin or cefoxitin for detection of methicillin-resistant Staphylococcus aureus. J Clin Microbiol 44:637-639

15. Warren DK, Liao RS, Merz LR, Eveland M, Dunne WM Jr (2004) Detection of methicillin-resistant Staphylococcus aureus directly from nasal swab specimens by a real-time PCR assay. J Clin Microbiol 42:5578-5581

16. Huletsky A, Lebel P, Picard FJ, Bernier M, Gagnon M, Boucher N, Bergeron MG (2005) Identification of methicillin-resistant Staphylococcus aureus carriage in less than 1 hour during a hospital surveillance program. Clin Infect Dis 40:976-981

17. Bishop EJ, Grabsch EA, Ballard SA, Mayall B, Xie S, Martin R, Grayson ML (2006) Concurrent analysis of nose and groin swab specimens by the IDI-MRSA PCR assay is comparable to analysis by individual-specimen PCR and routine culture assays for detection of colonization by methicillin-resistant Staphylococcus aureus. J Clin Microbiol 44:2904-2908

18. Van Nguyen JC, Kitzis MD, Ly A, Chalfine A, Carlet J, Ben Ali A, Goldstein F (2006) Detection of nasal colonization methicillinresistant Staphylococcus aureus: a prospective study comparing real-time genic amplification assay vs selective chromogenic media. Pathol Biol (Paris) 54:285-292

19. Desjardins M, Guibord C, Lalonde B, Toye B, Ramotar K (2006) Evaluation of the IDI-MRSA assay for detection of methicillinresistant Staphylococcus aureus from nasal and rectal specimens pooled in a selective broth. J Clin Microbiol 44:1219-1223

20. Oliveira DC, Tomasz A, Lencastre H de (2002) Secrets of success of a human pathogen: molecular evolution of pandemic clones of meticillin-resistant Staphylococcus aureus. Lancet Infect Dis $2: 180-189$ 